\section{Social cognition and face processing} in schizophrenia

JEREMY HALL, JONATHAN M. HARRIS, REINER SPRENGELMEYER, ANKE SPRENGELMEYER, ANDREW W. YOUNG, ISABEL M. SANTOS, EVE C. JOHNSTONE and STEPHEN M. LAWRIE

\begin{abstract}
Summary Studies of face processing have begun to elucidate the brain regions involved in social cognition, which include frontal and temporal regions known to be reduced in volume in schizophrenia. In this case-control study participants with schizophrenia $(n=20)$ showed marked deficits in their ability to interpret social cues from faces, and those experiencing positive symptoms were impaired in recognising even basic facial emotions.
\end{abstract}

\section{Declaration of interest None.}

Deficits in social function are characteristic of schizophrenia. Much social information is gathered by observing faces, and the interpretation of such information requires the interaction of brain regions specialised for processing different aspects of facial information (Adolphs, 2001). In particular, the recognition of emotion in faces requires the amygdala, insula and basal ganglia (Adolphs, 2001), whereas more complex social judgements are believed to depend upon an interaction between areas of prefrontal cortex and the temporal lobe (Adolphs, 2001; Winston et al, 2002). Many of these regions, including the amygdala and the prefrontal and superior temporal cortices, are reduced in volume in schizophrenia (Wright et al, 2000). However, although some previous studies have shown deficits in facial emotion recognition in schizophrenia, others have not, or have found more generalised impairments in facial processing (Mandal et al, 1998). Furthermore, as far as we are aware, there has been no previous study of the ability of patients with schizophrenia to make more complex social judgements.

\section{METHOD}

Participants underwent tests of facial identity recognition and facial emotion recognition and a novel test of social judgement from faces. Facial identity was tested using the Benton test of Facial Recognition, short form (described in Benton et al, 1983). Facial emotion recognition was tested using two tests in which subjects were required to identify which of six named emotions (happiness, surprise, fear, sadness, disgust or anger) was expressed in pictures of actors. In the first test (Ekman $60)$ the prototypical emotional expressions were shown, while in the second test (Hexagon) computer generated images morphed between emotions likely to be confused were used to provide a more stringent test of emotion recognition (tests described in Sprengelmeyer et al, 1996; Young et al, 2002).

More complex social judgements were assessed using a novel test (Social Cognition test). Five hundred pictures of faces derived from media sources, of non-famous adults, were rated by six volunteer participants on six social dimensions on a scale of 1-7. The end-points of each scale were, respectively, young or old; very untrustworthy or very trustworthy; very unattractive or very attractive; very typical or very distinctive; very unintelligent or very intelligent; very unapproachable or very approachable. The faces were highly reliably related on each dimension across all raters $(P<0.01)$. For each dimension, 40 faces were selected, comprising 20 faces representative of each valence, and these were used to construct the final test. The sets of faces for each social dimension were matched as closely as possible on the remaining five dimensions. In the test series participants were shown the six sets of 40 faces ( 8 practice and 32 test images). Faces were shown for $3 \mathrm{~s}$ each, and the response choices (for example, 'old' or 'young') were shown on the screen. Participants were told that faces were chosen to be half of each category. No feedback was given as to the appropriateness of any response. Responses were scored according to their agreement with the response most commonly selected in the pilot study, giving a maximum score of 32 .

\section{RESULTS}

Twenty patients meeting DSM-IV criteria for schizophrenia (American Psychiatric Association, 1994) participated in the study. Their mean age was 33.4 years (s.d.=12.3), mean premorbid IQ (Nelson \& Willison, 1991) was 111.1 (s.d.=8.1), and their mean antipsychotic dosage was $317 \mathrm{mg}$ chlorpromazine equivalents (s.d.=275). They were matched with 20 control participants (mean age 35.3 years, s.d.=11.4; mean IQ 113.8 , s.d.=6.2). Each group contained 10 men and 10 women. Symptoms in the patient group were rated immediately prior to testing using the Positive and Negative Syndrome Scale (PANSS; Kay et al, 1987) by a single clinician (J.H.). Exclusion criteria were age under 18 or over 65 years, organic brain disease, dependence on alcohol or non-prescribed drugs and history of any other major psychiatric disorder. Ethical approval was obtained from the Lothian Research Ethics Committee.

Patients showed no difference from the control group in performance on the Benton Test of Facial Recognition $(F(1,38)=0.02$, $P=0.9$ ), but participants with schizophrenia showed an overall deficit in facial emotion recognition on both the Ekman 60 $(F(1,38)=5.7, \quad P<0.05)$ and Hexagon $(F(1,38)=4.2, P<0.05)$ tests. Furthermore, the schizophrenia group showed a highly significant impairment relative to the control group across the tests of social cognition $(F(1,38)=34.7, P<0.001$; effect size 2.3$)$.

Previous studies had demonstrated a differential deficit in facial emotion recognition in patients experiencing positive symptoms (e.g. Kohler et al, 2000), so we examined this effect by subdividing the schizophrenia group into those with positive symptoms (those with a score of $\geqslant 4$ on a positive symptom item in the PANSS; $n=11$ ), and those without positive symptoms $(n=9)$. There was no difference between patients with and without positive symptoms on either the Benton Test of Facial Recognition or the Social Cognition test $(P>0.9$ for both, Fig. 1a). However, there were significant group differences on the Ekman $60(F(2,37)=6.1, P<0.01)$ and Hexagon $(F(2,35)=7.3, P<0.01)$ tests, which derived from a deficit in the positive symptoms group $(P<0.01)$, but not in the 
(a)

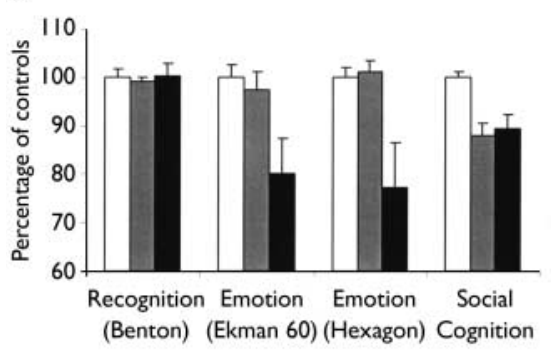

(b)

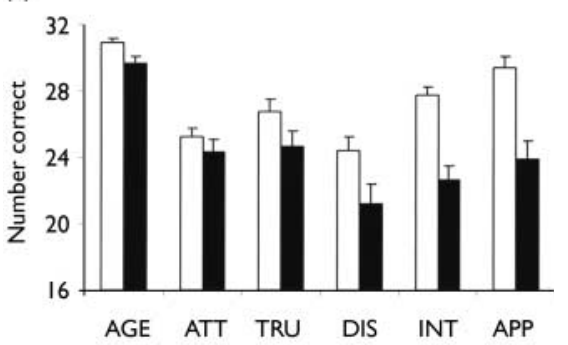

Fig. I (a) Overall performance of patients with positive symptoms of schizophrenia ( $\square)$ and patients without positive symptoms ( $\square$ ) on tests of facial identity recognition (Benton), facial emotion recognition (Ekman 60 and Hexagon) and social judgement from faces (Social Cognition). There was no difference between the patients with and without positive symptoms on baseline demographic measures. Bars show the mean number of correct responses for each test, expressed as a percentage of the level of correct responding in the control group (). (b) Performance of patients with schizophrenia (घ) and control participants ( $\square$ ) on tests of social judgement of faces previously rated for age (AGE), attractiveness (ATT), trustworthiness (TRU), distinctiveness (DIS), intelligence (INT) and approachability (APP). Bars show the mean number of correct responses for each test (maximum 32; chance level of responding, 16). Error bars show the s.e.m.

group without positive symptoms $(P>0.9)$, relative to controls (Fig. 1a).

Analysis of the performance of the patient and control groups across the six individual social cognition tests (Fig. 1b) showed a significant group $\times$ test interaction $(F(3,129)=4.0, P<0.01)$. Post hoc tests using Bonferroni corrections showed that the group with schizophrenia was particularly impaired on judgements of approachability $(P<0.001)$, intelligence $(P<0.001)$ and distinctiveness $(P<0.05)$.

Correlation analysis confirmed that none of the effects could be accounted for

JEREMY HALL, MB, PhD, JONATHAN M. HARRIS, BSc, Division of Psychiatry, School of Molecular and Clinical Medicine, University of Edinburgh; REINER SPRENGELMEYER, PhD, ANKE SPRENGELMEYER, MSc, School of Psychology, University of St Andrews; ANDREW W. YOUNG PhD, ISABEL M. SANTOS, PhD, Department of Psychology, University of York; EVE C. JOHNSTONE, FRCPsych, STEPHEN M. LAWRIE, MRCPsych, School of Molecular and Clinical Medicine, University of Edinburgh, UK

Correspondence: Dr Jeremy Hall, Psychiatry Department, Lynebank Hospital, Halbeath Road, Dunfermline, Fife KYII 4UW, UK

(First received 21 July 2003, final revision 22 December 2003, accepted 5 April 2004)

by variations in illness duration, dosage of antipsychotic or depression scores on the PANSS. Performance on the facial emotion recognition tasks, but not on the Social Cognition tests, correlated with anxiety ratings on the PANSS. Analysis of covariance confirmed that the deficits in social cognition remained significant after covarying for performance on the facial recognition tasks.

\section{DISCUSSION}

Patients with schizophrenia showed severe deficits in their ability to make social judgements from facial expressions, and participants experiencing positive symptoms were impaired in recognising even basic facial emotions. These results could not be accounted for in terms of a general impairment in facial information processing, as all participants were able to recognise facial identity normally.

Making complex social judgements from faces requires interaction between the frontal and temporal cortices (Adolphs, 2001; Winston et al, 2002). These areas are known to be structurally abnormal in schizophrenia, and disconnectivity between these regions is implicated in the disease (Wright et al, 2000; Burns et al, 2003). Our findings suggest that impairments in social cognition represent a core deficit in schizophrenia, which is likely to be related to abnormal frontotemporal function. In addition, the inability of patients with positive symptoms to identify even basic facial emotions suggests that the brain regions involved in facial emotion recognition, such as the amygdala, are subject to functional impairment during psychotic episodes, or that mechanisms of compensation for deficits in these regions fail when subjects develop positive symptoms. These deficits in social function and emotion recognition are of clinical importance both in terms of patients' vulnerability in social interactions and in the design of psychosocial treatments.

\section{ACKNOWLEDGEMENTS}

The study was funded by the Stanley Medical Research Institute. We thank Sophia Durrani for help in preparing the social cognition test.

\section{REFERENCES}

Adolphs, R. (200I) The neurobiology of social cognition. Current Opinion in Neurobiology, II, 231-239.

American Psychiatric Association (1994) Diagnostic and Statistical Manual of Mental Disorders (4th edn) (DSM-IV). Washington, DC: APA.

Benton, A. L., Hamsher, K. S., Varney, N., et al (1983) Contributions to Neuropsychological Assessment: A Clinical Manual. Oxford: Oxford University Press.

Burns, J., Job, D., Bastin, M. E., et al (2003) Structural disconnectivity in schizophrenia: a diffusion tensor magnetic resonance imaging study. British Journal of Psychiatry, 182, 439-443.

Kay, S. R., Fiszbein, A. \& Opler, L. A. (1987) The positive and negative syndrome scale (PANSS) for schizophrenia. Schizophrenia Bulletin, I3, 26I-276.

Kohler, C. G., Bilker, W., Hagendoom, M., et al (2000) Emotion recognition deficit in schizophrenia: association with symptomatology and cognition. Biological Psychiatry, 48, $127-136$

Mandal, M. K., Pandey, R. \& Prasad, A. B.

(1998) Facial expression of emotions and schizophrenia: a review. Schizophrenia Bulletin, 24, 399-412.

Nelson, H. E. \& Willison, J. R. (1991) The Revised National Adult Reading Test - Test Manual.Windsor: NFER-Nelson

Sprengelmeyer, R., Young, A.W., Calder, A. J., et al (1996) Loss of disgust: perception of faces and emotions in Huntington's disease. Brain, I19, 1647-1665.

Winston, J. S., Strange, B. A. \& O’Doherty, J. (2002) Automatic and intentional brain responses during evaluation of trustworthiness of faces. Nature Neuroscience, 5, 277-283.

Wright, I. C., Rabe-Hesketh, S., Woodruff, P. W. R. et al (2000) Meta-analysis of regional brain volumes in schizophrenia. American Journal of Psychiatry, 157, 16-25.

Young, A. W., Perrett, D., Calder, A., et al (2002) Facial Expressions of Emotion: Stimuli and Test. Bury St Edmunds: Thames Valley Test Co. 